\title{
Genetic Algorithms for Municipal Solid Waste Collection and Routing Optimization
}

\author{
Nikolaos V. Karadimas, Katerina Papatzelou and Vassili G. Loumos \\ National Technical University of Athens, \\ School of Electrical and Computer Engineering, \\ Multimedia Technology Laboratory, \\ Heroon Polytechneiou 9, Zografou Campus, 15780 Athens, Greece \\ e-mail:nkaradim@central.ntua.gr, rina310@gmail.com, loumos@cs.ntua.gr
}

\begin{abstract}
In the present paper, the Genetic Algorithm (GA) is used for the identification of optimal routes in the case of Municipal Solid Waste (MSW) collection. The identification of a route for MSW collection trucks is critical since it has been estimated that, of the total amount of money spent for the collection, transportation, and disposal of solid waste, approximately $60-80 \%$ is spent on the collection phase. Therefore, a small percentage improvement in the collection operation can result to a significant saving in the overall cost. The proposed MSW management system is based on a geo-referenced spatial database supported by a geographic information system (GIS). The GIS takes into account all the required parameters for solid waste collection. These parameters include static and dynamic data, such as the positions of waste bins, the road network and its related traffic, as well as the population density in the area under study. In addition, waste collection schedules, truck capacities and their characteristics are also taken into consideration. Spatiotemporal statistical analysis is used to estimate inter-relations between dynamic factors, like network traffic changes in residential and commercial areas. The user, in the proposed system, is able to define or modify all of the required dynamic factors for the creation of alternative initial scenarios. The objective of the system is to identify the most cost-effective scenario for waste collection, to estimate its running cost and to simulate its application.
\end{abstract}

\section{Introduction}

Nowadays, waste management is one of the main environmental and socio-economic problems, especially in towns and large cities which are densely populated. The rapid and constant growth in urban population led to a dramatic increase in urban

Please use the following format when citing this chapter:

Karadimas, N. V., Papatzelou, K., Loumos, V. G., 2007, in IFIP International Federation for Information Processing, Volume 247, Artificial Intelligence and Innovations 2007: From Theory to $\Lambda$ pplications, eds. Boukis, C., Pnevmatikakis, 1., Polymenakos, 1.., (Boston: Springer), pp. 223-231. 
solid waste production, which in turn forced many municipalities to assess their solid waste management program and examine its environmental and cost-effectiveness in terms of collection, transportation, processing and landfill.

The management of urban solid waste is a hard task since it is necessary to take into account conflicting objectives. Therefore, a multi-criteria urban waste management decision approach is needed. In order for the municipalities to implement sustainable management of urban solid waste, more comprehensive use of data collection and information systems is required. Information technology (IT) gave the development of information systems in waste management a boost. Direct database accessing, in combination with GIS-techniques, provide relevant information for visualization, planning and decision making of regional waste collection (for example planning routes, and forecasting the amount of needed trucks).

The identification of a route for MSW collection trucks is critical since it has been estimated that, of the total amount of money spent for the collection, transportation, and disposal of solid waste, approximately $60-80 \%$ is spent on the collection phase [1]. Therefore, a small percentage improvement in the collection operation can result to a significant saving in the overall cost.

The present paper mainly focuses on the collection and transport of solid waste from any loading spot in the area under study. In addition, other factors that affect the whole system will be mentioned and discussed. Of course, this research covers only the routes included in the given area, but can be applied to any other area, simply by changing the input data. Therefore, a framework (schema) for the design and implementation of a solution for the solid waste collection and routing is proposed. According to this schema, the genetic algorithm is introduced and implemented, for defining the optimal collection route. In the end, the results of the GA are also compared with the empirical method that the Municipality of Athens (MoA) uses to collect and transport the solid waste to the landfill site.

This scheme is described in the rest of the paper, first the theoretical and methodological aspects for urban solid waste management and the related work that has been done in this area is described. This is followed by an introduction to the waste management problem in the selected case study area. Thereafter the GA optimization algorithm is introduced and implemented for the area under study. The results of the GA algorithm and its comparison with the present empirical solution that the MoA uses are illustrated and conclusions are discussed.

\section{Related Work}

In the literature, many methods and algorithms have been used for optimizing routing aspects of solid waste collection networks. Many papers have modeled the optimization problem of urban waste collection and transport as various versions of the Arc Routing Problem (ARP) [2], [3], [4]. Nevertheless, the particular problem has been also modeled as a Vehicle Routing Problem (VRP) in which a set of waste trucks have to serve a set of waste bins minimizing the cost function while being subject to a number of constraints. The characteristics of the vehicles and of the 
constraints determine the various types of VRPs [5], [6], [7]. However, the speed distributions, from which the travel times can be calculated, are supposed to be known at the beginning of the optimization.

In addition, the routing problem of the solid waste collection process has been treated as a Traveling Salesman Problem (TSP), which can be formulated as finding the minimum of a function representing the route that visits all the nodes of a set, at least once. An Ant Colony System (ACS), a distributed algorithm inspired by the observation of real colonies of ants, has been presented [8], [9], for the solution of TSP and ATSP problems [10], [11]. Viotti et al. [12] introduce a Genetic Algorithm for solving the TSP and present its results. Poser and Awad [13] have developed a methodology based on real genetic algorithm for effectively solving the TSP in the field of solid waste routing system in the large cities.

In this context the problem is reduced to a Single Vehicle Origin Round trip Routing which is similar to the common Traveling Salesman Problem (TSP). The well-known combinatorial optimization problem, in which each waste truck, in this work, is required to minimize the total distance traveled to visit, only once, all the waste bins in its visiting list. In the traditional TSP, the cost of traveling (distance) between two waste bins does not depend on the direction of travel. Hence, the cost (distance) matrix representing the parameters of the problem is symmetric. However, the problem, which this work refers to, is modeled as an Asymmetric TSP (ATSP) problem due to road network restrictions. An ATSP problem considers that the bidirectional distances between a pair of waste bins are not necessarily identical. The ATSP problem can be solved to optimality using various algorithms. Some heuristic procedures for solving the symmetric version of the problem (TSP) can be easily modified for the asymmetric versions, but others are not. The objective of this paper is the application of a Genetic Algorithm for the identification of optimal routes in the case of Municipal Solid Waste (MSW) collection.

\section{Case Study}

In this research work, a small part of Attica's prefecture (a suburb of Athens) was chosen as the case study area. The MoA has empirically divided its area in about 122 solid waste collecting programs, where in general each one includes approximately 100 waste bins. Any garbage truck that is responsible for the collection of the solid waste in that given area must visit all the bins in order to complete its collection program.

This examined area is about $0.45 \mathrm{Km}^{2}$, with a population of more than 8,500 citizens and a production of about 3,800 tones of solid urban waste per year. The data concerning the area under examination was obtained from the pertinent Agency of the MoA. This data includes maps of the examined area with the correspondent annotation (address and numbering labels of streets), the building blocks and the locations of the existing waste bins. The waste bins locations were initially derived from a pilot program that the MoA was using for the allocation of their trucks.

Fig 1 illustrates the loading spots in the area under study (totally 72), in which each loading spot may represent one or more waste bins. The location of these 
loading spots was defined by the MoA to serve the needs of the present waste collection system. A different placement of the loading spots would assist the proposed model better but this issue is beyond the scope of the present paper. A method for optimal placement of waste bins is described in Karadimas et al. [15].

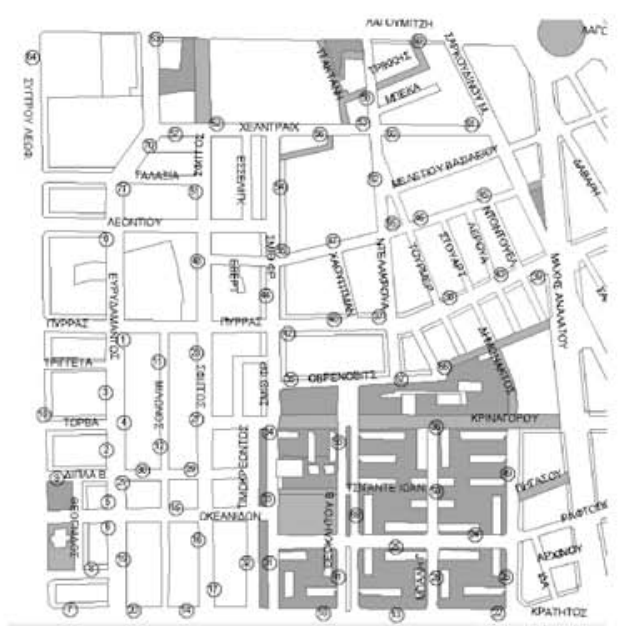

Fig. 1. The 72 dots, in the map of the study area, represent the loading spots that the Municipality presently uses.

Finally, the information stored in the aforementioned database such as the present sequence of the waste collection and transport that the Municipality uses (called the empirical method), has been compared to the derived results of the proposed algorithms in the following sections.

According to the above, the urban solid waste collection and transport is a complex problem with many limitations. Minimization of cost means minimization of collection time and not necessarily choosing the minimal route. In addition, each waste truck is able to collect a specific quantity of solid waste due to its limited waste capacity. So, the collected area, considering all parameters for that part of the problem, should be fragmented to sub programs, in which the produced quantity of solid waste is equal to or less than the capacity of each truck. Therefore, the problem in our case, as mentioned above, can be classified as a Asymmetric Traveling Salesman Problem (ATSP): "Given a set of $\mathrm{n}$ loading spots and the transport cost between any loading spots, the ATSP can be stated as the problem of finding a minimal cost closed tour that visits each loading spot only once and bi-directional distances between at least one pair of loading spots are not necessarily identical". 


\section{Genetic Algorithm Implementation}

Genetic Algorithms (GA) were invented by John Holland [14] and his colleagues in the early 1970s and are inspired by Darwin's theory of evolution. GA use genetic operators such as selection, crossover, and mutation for the exploration of solutions to complex problems. Initially, GA randomly yields a population (set of candidate solutions or individuals). Each individual is characterized by certain fitness. In each iteration, a selection of individuals from the entire population takes place according to their fitness values and then the chosen solutions are perturbed (through the mechanisms of crossover or mutation) in order to yield a new generation. This process is repeated for a number of iterations until a predefined termination criterion is satisfied.

In the following paragraphs, the GA implementation is presented for the under study optimization problem. The initial population is randomly produced by GA implementation. Fig 2 illustrates a snapshot of the GUI for our GA implementation. The candidate solutions which comprise the population are represented as a list showing the order in which the loading spots (nodes) are visited by the waste truck.

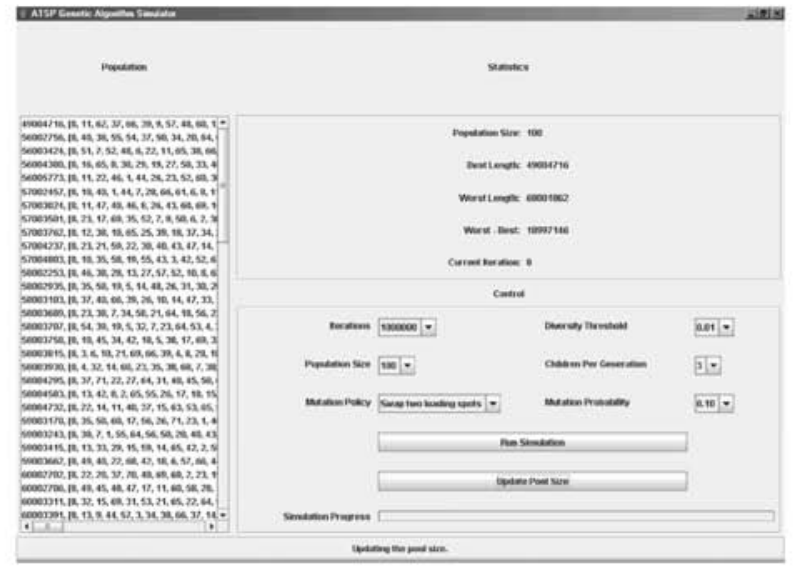

Fig. 2. The Graphical User Interface for the Genetic Algorithm implementation

The objective function is the minimization of the total tour length of the waste truck through a set of loading points. The population in the GA program evolves from one generation (iteration) to the next through the use of genetic operators. Three genetic operators are used in the GA program: Selection, Crossover and Mutation.

During the selection operation, the algorithm selects one parent from the top half of the candidate solutions (population) and the other parent from anywhere inside the population. This tends to slightly favor the "fitter" individuals since the candidate solutions of the population are sorted by tour length.

The operation of crossover selects two loading spots from inside a tour. Next, the sub-tour of one of the parents (between these two points) is copied directly to the 
child. The remaining loading spots in the tour are places around the child's sub-tour in the same order that they occur in the other parent.

Finally, in the mutation phase, two different functions are used. The user can select the mutation operator of his preference through the user interface. The first method simply reverses a random sub-tour in a given tour. The second randomly selects two loading spots and swaps their positions. Through experimentation, it was discovered that that the second mutation mechanism yielded better results than the first one. To sum up, it is worth mentioning that the values for all the parameters of the GA (iterations, population size, mutation policy, mutation probability, diversity threshold and children per generation) are user configurable and good parameter tuning is essential for the GA to produce satisfactory solutions.

\section{Results of Genetic Algorithm}

In order to execute the GA, the user should adjust the values of the following six parameters: Iterations, Population, Children per Generation, Mutation Policy, Mutation Probability and Diversity Threshold. The values of the parameters of this algorithm can be selected from the following set of values:

- Iterations $\in\{100,000,1,000,000\}$,

- Population $\in\{20,50,72,100,144,300\}$,

- Children per Generation $\in\{1,2,3,4,5\}$,

- Mutation Policy $\in$ \{Swap two loading spots, Reverse sub tour\},

- Mutation Probability $\in\{0,0.05,0.1,0.2,0.4,0.8\}$ and

- Diversity Threshold $\in\{0,0.01,0.05,0.1,0.5\}$

In order to cover all the possible solutions that the aforementioned parameter settings can produce, there are 3,600 different combinations, which make the testing process even more difficult. The set of values for the GA parameters presented above is common in the literature and this is why they have chosen. Through the process of trial and error the GA has been executed more than 1,200 times in order to find the best parameters values combination. Table 1 presents the total number of the GA algorithm execution and the acceptable solution (solutions which their values are less than $1,000,000)$ as well as the number of the solutions which are better than the empirical one. Besides Fig 3 illustrates the distribution of these solutions (accepted and not accepted) yielded by the GA.

Table 1. The analysis of experimental resu lts for GA.

\begin{tabular}{|c|c|c|c|c|}
\hline \multirow{2}{*}{ Num of runs } & \multirow{2}{*}{ Not accepted } & \multicolumn{3}{|c|}{ Accepted } \\
\cline { 3 - 5 } & & Better & Worse & Total \\
\hline 12,000 & 10,200 & 450 & 1,350 & 1,800 \\
\hline
\end{tabular}




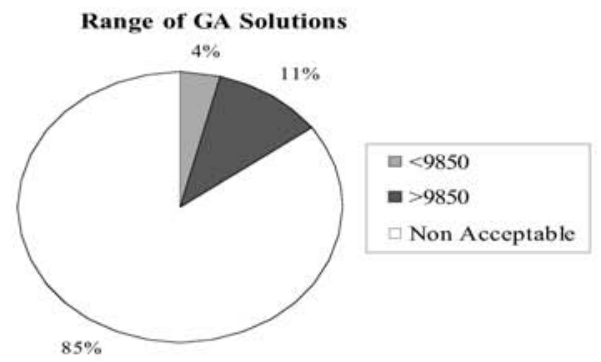

Fig. 3. A pie depicting the range of the solutions yielded by GA.

Eventually, the best result of this algorithm stemmed from the following parameter setting:

- Iterations: 200,000,

- Population: 100 ,

- Children per Generation: 5,

- Mutation Policy:

- Swap 2 Loading Spots - the $1^{\text {st }} 100,000$ iterations $(9,084)$ and

o Reverse Subtour - the $2^{\text {nd }} 100,000$ iterations $(8,902)$,

- Mutation Probability: 0.1,

- Diversity Threshold: 0.01 .

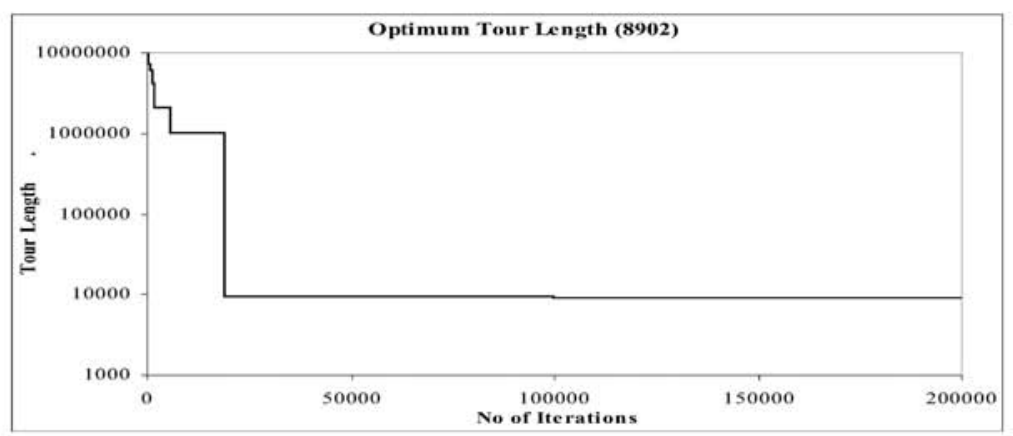

Fig. 4. The best result of the Genetic Algorithm.

Fig 4 illustrates the graphical representation of the best result of GA execution $(8,902)$. The algorithm converges in the first 21,000 iterations and in the following iterations only a slight improvement is achieved. In general, a considerable number of iterations are required in order for the GA to yield satisfactory solutions. Subsequently, the computation time required is sizeable and this was detrimental to the testing. Practically, the algorithm converges at the final optimum solution in 
approximately 15,000 iterations. Therefore, the algorithm produces good results from the first $10 \%$ of the algorithm executions even for the experiments that have been executed for $2,000,000$ iterations. That is the reason that the executions of the algorithm have been arranged at 100,000 iterations.

Concluding, Table 2 illustrates the best solution, that GA produce, which has a significantly improvement $(9.62 \%)$ in comparison to the respective of the empirical method which is currently used by MoA $(9,850)$.

Table 2. The comparison between the best GA solution and the empirical model

\begin{tabular}{|c|c|c}
\hline $\begin{array}{c}\text { Empirical } \\
\text { Model (meter) }\end{array}$ & $\begin{array}{c}\text { GA Optimized } \\
\text { route (meter) }\end{array}$ & $\begin{array}{c}\text { Improvement } \\
(\%)\end{array}$ \\
\hline 9,850 & 8,902 & $9.62 \%$ \\
\hline
\end{tabular}

\section{Conclusions}

This work focuses on the collection and transport of solid waste from waste bins in the area under study. The Genetic Algorithm is introduced and implemented, for monitoring, simulation, testing, and route optimization of alternative scenarios for a solid waste management system.

The first experiments have shown that applying the GA for the solution of this every day problem - the collection of urban solid waste - can greatly minimize the collection tour length and eventually the total cost in time and money. However, as it was reported above, the particular problem is much more complicated than presented in the current work. The proposed methodology was applied in a region of the MoA which contains a quantity of solid waste equal to the capacity of the waste truck used in this particular area. Therefore, the problem was transformed into a classic TSP problem.

Although the case study covers an area of about $0.45 \mathrm{~km}^{2}, 8,500$ citizens and over 100 building blocks, to ensure the reliability of the derived results, a future prospect of this work is that the proposed model should be tested in an even more extended area.

\section{References}

1. Municipality Of Athens. Estimation, Evaluation and Planning Of Actions for Municipal Solid Waste Services During Olympic Games 2004. Athens, Greece. (2003)

2. V. Maniezzo, Algorithms for large directed CARP instances: urban solid waste collection operational support, Technical Report UBLCS-2004-16, (2004).

3. S.K. Amponsah and S. Salhi, The investigation of a class of capacitated arc routing problems: the collection of garbage in developing countries, Waste Management 24, 711$721,(2004)$.

4. G. Ghiani, F. Guerriero, G. Improta and R. Musmannod, Waste collection in Southern Italy: solution of a real-life arc routing problem, Intl. Trans. in Op. Res. 12, 135-144, (2005). 
5. S. Sahoo, S. Kim and B.I. Kim, Routing Optimization for Waste Management, Interfaces Journal (SCIE), Informs (OR/MS), 35(1), 24-36, (2005).

6. B. Bullnheimer, R.F. Hartl, and C. Strauss, Applying the ant system to the vehicle routing problem. In: Osman, I.H., Voss, S., Martello, S. \& Roucairol C. (eds): Meta-Heuristics: Advances and Trends in Local Search Paradigms for Optimization, (Kluwer Academic Publishers, Dordrecht, The Netherlands, 1998), pp. 109-120.

7. A.V. Donati, R. Montemanni, N. Casagrande, A.E. Rizzoli, and L.M. Gambardella, Time-dependent Vehicle Routing Problem with a Multi Ant Colony System, Technical Report TR-17-03, IDSIA, Galleria 2, Manno, Switzerland (2003).

8. M. Dorigo, V. Maniezzo and A. Colorni, The ant system: optimization by a colony of cooperating agents, IEEE Transactions on Systems, Man and Cybernetics, 26(1), 1-13, (1996).

9. M. Dorigo and L.M. Gambardella, Ant Colony System: A Cooperative Learning Approach to the Traveling Salesman Problem, IEEE Transactions on Evolutionary Computation, 1(1) (1997).

10. N.V. Karadimas, G. Kouzas, I. Anagnostopoulos and V. Loumos, Urban Solid Waste Collection and Routing: The Ant Colony Strategic Approach. International Journal of Simulation: Systems, Science \& Technology, 6(12-13), 45-53, (2005).

11. N.V. Karadimas, K. Papatzelou and V.G. Loumos, Optimal solid waste collection routes identified by the ant colony system algorithm, Waste Management \& Research 25, 139147, (2007).

12. P. Viotti, A. Pelettini, R. Pomi and C. Innocetti, Genetic algorithms as a promising tool for optimisation of the MSW collection routes, Waste Management Research, 21, 292298, (2003).

13. I. von Poser, A.R. Awad, Optimal Routing for Solid Waste Collection in Cities by Using Real Genetic Algorithm, Information and Communication Technologies, ICTTA '06, 1, 221-226, (2006).

14. J.H. Holland, Adaptation in Natural and Artificial Systems: An Introductory Analysis With Applications to Biology, Control, and Artificial Intelligence, MIT Press, 1992. First Published by University of Michigan Press 1975.

15. N.V. Karadimas, O. Mavrantza and V. Loumos, GIS Integrated Waste Production Modelling. IEEE EUROCON 2005- The International Conference on "Computer as a Tool”, Belgrade, 22-24 November, pp. 1279-1282. Serbia \& Montenegro, (2005). 